\title{
Anterior Cruciate Ligament Reconstruction Using Bone-Patellar Tendon-Bone Allograft in Patients Aged 50 and Older Leads to Improved Activity Levels and Acceptable Patient-Reported Outcomes
}

\author{
Stephen M. Sylvia, M.D., Thomas J. Gill, M.D., Ian D. Engler, M.D., Kaitlin M. Carroll, B.S., \\ and Matthew J. Salzler, M.D.
}

Purpose: To evaluate patient-reported outcomes in patients aged 50 years and older undergoing anterior cruciate ligament reconstruction (ACLR) using bone-patellar tendon-bone (BPTB) allograft with minimum 2-year follow-up. Methods: A retrospective review was performed on a consecutive series of patients aged 50 and older who underwent ACLR using BPTB allograft by a single surgeon with minimum 2-year follow-up. Postoperative International Knee Documentation Committee (IKDC), Lysholm, and Physical Component Summary of the 12-item Short-Form Health Survey were used to assess outcomes, as well as preoperative and postoperative Tegner activity scores, which were compared using a paired sample $t$ test. Results: Fifty patients met inclusion criteria, with a mean age of $55.3 \pm 4.4$ years and mean follow-up of $4.8 \pm 1.9$ years. Tegner activity scores improved from a mean preoperative score of 3.26 to a mean postoperative score of $5.25(P<.001)$. The mean postoperative scores for Lysholm, IKDC, and Physical Component Summary were $87.3,81.1$, and 54.3 , respectively. In total, $36(72 \%)$ patients achieved a patient acceptable symptom state score for IKDC and $37(74 \%)$ patients achieved a minimal clinically important difference for Tegner activity score. Thirtyeight $(76 \%)$ patients reported good-to-excellent results, $6(12 \%)$ patients reported fair results, and $6(12 \%)$ patients reported poor results. Conclusions: ACLR with BPTB allograft in patients aged 50 and older leads to good patientreported outcomes with significantly increased postoperative activity status at a minimum 2-year follow-up. Level of Evidence: Level IV, therapeutic case series.

A nterior cruciate ligament (ACL) tears are the most common ligamentous injury, with an annual incidence of 68.6 per 100,000 person-years. ${ }^{1}$ The ACL

From the Department of Orthopaedics, Tufts Medical Center, Boston (S.M.S., I.D.E., M.J.S.); and Boston Sports Medicine, Dedham (T.J.G.), Massachusetts; and Hospital for Special Surgery, New York, New York (K.M.C.), U.S.A.

The authors report the following potential conflicts of interest or sources of funding: T.J.G. is a shareholder in VisionScope Technologies. K.M.C. reports stock options with OrthAlign, is a consultant for OrthAlign, and is an employee of Canary Medical. M.J.S. is a member of AANA. Full ICMJE author disclosure forms are available for this article online, as supplementary material.

Received February 8, 2021; accepted September 17, 2021.

Address correspondence to Matthew J. Salzler, M.D., Tufts Medical Center, Biewend Building, 7th Floor, 800 Washington St., Box 306, Boston, MA 02111.E-mail: MSalzler@tuftsmedicalcenter.org

(C) 2021 THE AUTHORS. Published by Elsevier Inc. on behalf of the Arthroscopy Association of North America. This is an open access article under the CC BY-NC-ND license (http://creativecommons.org/licenses/by-nc-nd/4.0/). 2666-061X/21207

https://doi.org/10.1016/j.asmr.2021.09.018 is essential for normal knee kinematics, and injury to the ACL may result in subsequent meniscal damage, recurrent instability, and articular cartilage pathology across all age groups. ${ }^{2-4}$ Outcomes following anterior cruciate ligament reconstruction (ACLR) are well studied and documented, although the majority of ACLR studies predominately focus on patients younger than 50 years old. ${ }^{5-7}$

However, due in part to improved surgical techniques and rehabilitation protocols, the incidence of ACLR in patients older than 50 years is increasing. ${ }^{8}$ In recent years, there is also a growing body of literature on the outcomes of ACLR in older patients, although most reported cohorts include multiple reconstruction techniques or have low numbers of bone-patellar tendon-bone (BPTB) allograft cases. $^{9-14}$ Allograft BPTB graft may provide the benefits of less operative time and no donor-site morbidity. Additionally, BPTB may result in improved graft strength ${ }^{15}$ and incorporation time when compared with other allograft options in rabbit models. ${ }^{16}$ 
The purpose of this study was to evaluate patientreported outcomes (PROs) in patients aged 50 years and older undergoing ACLR using BPTB allograft with minimum 2-year follow-up. The hypothesis was that postoperative Tegner activity scores would improve with a minimal clinically important difference (MCID) compared with preoperative values and that the majority of patients would achieve a patient acceptable symptom state (PASS) for International Knee Documentation Committee (IKDC) score.

\section{Methods}

Following institutional review board approval, a consecutive series of patients aged 50 and older who underwent ACLR using BPTB allograft by a single fellowship-trained surgeon in the United States were retrospectively reviewed. Study inclusion criteria were age 50 years or older at the time of surgery, ACLdeficient knee, 1 or more episodes of knee instability, reconstruction using BPTB allograft, complete PROs, and minimum of 2 years' follow-up. Patients were excluded if concurrent ligamentous or bony procedures were performed. ACL tear was diagnosed based on subjective knee instability, positive Lachman test, and magnetic resonance imaging confirmation of a ruptured ACL. Decision to undergo ACLR was a shared decision with the patient, given their desired activity level, preinjury knee symptomatology, physiologic age, and degree of degenerative changes present on radiographs. Kellgren-Lawrence grade 4 osteoarthritis was a contraindication to ACLR, whereas grade 3 was a relative but not strict contraindication depending on the patient. All patients engaged in a structured physical therapy rehabilitation protocol to regain their range of motion (ROM) preoperatively. Charts were reviewed to identify patient demographics, surgical procedure, perioperative complications, and reports of retear or clinical failure. All patients were contacted by mail and telephone after a minimum of 2 years postoperatively to collect outcome measures. PROs included preoperative and postoperative Tegner activity score and postoperative IKDC, Lysholm, and Physical Component Summary of the 12-item ShortForm Health Survey. The PASS score for IKDC was set at 75.9, and MCID for Tegner activity score was set at 1 , in accordance with literature standards. ${ }^{17,18} \mathrm{~Pa}$ tients were categorized based on Lysholm score, with excellent results between 95-100, good results between 84-94, fair results between 65-83, and poor results less than $65 .{ }^{19}$ Preoperative and postoperative Tegner scores were compared using a paired sample $t$ test performed with a statistical significance set at $P<.05$ and using the XL Stat PRO software package (New York, NY).

\section{Surgical Technique}

Anesthesia included general anesthesia and a femoral nerve block. All patients underwent an examination under anesthesia for ROM and stability testing including Lachman, pivot shift, anterior and posterior drawer, and dial test to confirm anterior joint subluxation and to evaluate for concurrent ligamentous injury. The BPTB allograft was prepared to fit through a 10-mm spacer on the back table with $25-$ to $35-\mathrm{mm}$ long bone blocks. The graft was taken from the center of the patellar tendon. A diagnostic knee arthroscopy was then performed, and concomitant intra-articular pathology was identified and addressed. Using a tibial guide set at 55 degrees, a $10-\mathrm{mm}$ tibial tunnel was established. A 10-mm transtibial femoral tunnel was then drilled, in accordance with described techniques which recreate the anatomic footprint. ${ }^{20,21}$ The graft was passed and fixed in full extension with bioabsorbable interference screws in all patients. All grafts were sterilized using MTF and LifeNet Allowash solution.

\section{Postoperative Rehabilitation Protocol}

All patients underwent a standard postoperative ACLR allograft protocol at the treating physician's office. This protocol consisted of 5 phases to protect the reconstructed ligament and ease patients back into activity with an emphasis on early ROM. Patients were partial weight-bearing for 6 weeks with ROM between 0 and $90^{\circ}$ allowed in a hinged knee brace before weaning to weight-bearing and ROM as tolerated. Heel slides and quadriceps sets were started in the immediate postoperative period, stationary bike was initiated at postoperative week 2 , and single-leg strengthening was initiated at postoperative week 6 . Patients could return to most strenuous job-related activities in 4 to 6 months. Return to running was permitted at 4 months with a custom-fit brace, with no restrictions at 6 months as long as strength and motion had been restored. All patients continued brace use for 1 year postoperatively.

\section{Results}

Fifty patients qualified for inclusion with a mean age of $55.3 \pm 4.4$ years and mean follow-up of $4.8 \pm 1.9$ years, with $28(56 \%)$ patients being female (Table 1$)$. Tegner activity scores improved from a mean preoperative score of $3.26 \pm 2.0$ to a mean postoperative score of $5.25 \pm 1.5(P<.001)$. The mean postoperative scores for IKDC, Lysholm, and Physical Component Summary were $81.1 \pm 16.1,87.3 \pm 15.1$, and $54.3 \pm 6.5$, respectively. In total, $36(72 \%)$ patients achieved a PASS score for IKDC and $37(74 \%)$ patients achieved a MCID for Tegner activity score. Based on Lysholm scores, $24(48 \%)$ patients reported excellent results, $14(28 \%)$ reported good results, $6(12 \%)$ reported fair 
Table 1. Patient Demographics and Functional Outcomes

\begin{tabular}{lc}
\hline & Values \\
\hline Number of patients, n & 50 \\
Age at surgery, y, mean (SD) & $55.3(4.4)$ \\
Length of follow-up, y, mean (SD) & $4.8(1.9)$ \\
Female sex, n (\%) & $28(56 \%)$ \\
Preoperative Tegner, mean (SD) & $3.26(2)^{*}$ \\
Postoperative Tegner, mean (SD) & $5.25(1.5)^{*}$ \\
IKDC, mean (SD) & $81.1(16.1)$ \\
Lysholm, mean (SD) & $87.3(15.1)$ \\
SF-12 Physical, mean (SD) & $54.3(6.5)$ \\
SF-12 Mental, mean (SD) & $51.8(8.1)$ \\
\hline
\end{tabular}

IKDC, International Knee Documentation Committee; SD, standard deviation; SF, short form.

${ }^{*} P<.001$, paired sample $t$ test.

results, and $6(12 \%)$ reported poor results (Table 2$)$. There were no reported clinical failures or surgical complications, including postoperative infections, deep venous thromboses, or further surgical procedures.

\section{Discussion}

We found good patient-reported clinical outcomes of ACLR with BPTB allograft in patients aged 50 years and older at mean 5-year follow-up. Tegner activity scores significantly improved postoperatively. The majority of patients reported good-to-excellent results, similar to previous studies evaluating other techniques. ${ }^{22,23}$ The present dataset represents a large cohort of ACLR using entirely BPTB allograft for this age group with validated outcome scores. These results suggest that patients older than 50 years can expect improved function and good-to-excellent outcomes from ACLR using BPTB allograft with minimal complications.

We believe BPTB allograft is an excellent graft choice in this population, given the faster surgical recovery, decreased donor-site morbidity, less operative time, less postoperative pain, and decreased risk of patellar tendon rupture or patellar fracture associated with allografts when compared to autografts. ${ }^{24,25}$ Although failure rates have been shown to be greater with allograft than autograft reconstruction, studies have suggested that this finding may be age-dependent, with some registries suggesting equivalent re-tear rates in older patients. ${ }^{25-27}$ When selecting among allografts, there is limited support in the literature given that allografts are generally grouped together. Our preference for BPTB allograft stems from its low rate of failure, biomechanical properties, and strength of fixation. ${ }^{28}$ Bone-to-bone healing has also been suggested to be superior to soft tissue-to-bone healing with regards to graft strength ${ }^{15}$ and incorporation time in a rabbit model. ${ }^{16}$ Furthermore, evidence of the limitations of soft tissue allografts has been reported. ${ }^{29}$

There are few studies that evaluate BPTB allografts in patients 50 years of age and older. Dahm et al. ${ }^{30}$ studied
34 patients ( 35 knees) older than the age of 50 who underwent ACLR, 23 with BPTB allograft and 12 with BPTB autograft. Postoperatively, only 2 knees $(6 \%)$ had a positive Lachman, and postoperative improvement was seen in IKDC, Lysholm, and University of California Los Angeles activity scores. Good outcomes have been shown in several other studies of ACLRs in older patients using various grafts, with the most commonly studied grafts being hamstring allograft and autograft. ${ }^{12,13,23,31-33}$ Fayard et al. ${ }^{33}$ investigated outcomes of ACLR in a multicenter retrospective study of 398 patients older than the age of 50 years. Their cohort consisted of $32 \%$ BPTB grafts and $68 \%$ hamstring grafts, although they did not specify the proportion of allograft and autograft. In total, $83 \%$ of their patients returned to sport, and significant improvements were noted in laxity as well as clinical outcome scores. A recent systematic review of ACLR in patients older than 50 years old found good results with all graft types, although only 65 patients ( $13.8 \%$ of their population) had BPTB allograft, and they did not stratify results by allograft source. $^{34}$

When considering ACLR in the older population, nonoperative treatment must certainly be considered. While many patients aged 50 and older may have acceptable function with a torn ACL, studies have shown that age alone is not a contraindication to ACLR. ${ }^{34}$ Additionally, more important than chronologic age is physiologic age. Surgeons must select motivated patients desiring return to high levels of activity when considering an ACLR in this age group.

\section{Limitations}

There are several limitations to this study. The retrospective design enables subject response and recall bias. The surgeries were performed by a single surgeon, which limits the generalizability of the study. There is no comparison group of different graft types, different age groups, or nonoperative treatment, which would help place our data in context. Further data on preinjury outcome scores would have allowed more indepth analysis of patients' return to their baseline functional level. Additionally, given that only patients with complete PROs were included, there could be a component of selection bias. There was no standardized method for addressing additional intra-articular pathology. Finally, our measured outcomes consist of

Table 2. Lysholm Score Categories

\begin{tabular}{lr}
\hline \multicolumn{1}{c}{ Score (points) } & $\mathrm{N}(\%)$ \\
\hline Excellent (95-100) & $24(48 \%)$ \\
Good $(84-94)$ & $14(28 \%)$ \\
Fair $(65-83)$ & $6(12 \%)$ \\
Poor $(<65)$ & $6(12 \%)$ \\
\hline
\end{tabular}


subjective PROs without objective data. This particularly complicates clinical failures, or retears. This population may not be aware of a retear several years postoperatively, given that demand on the knee tends to decrease with age.

\section{Conclusions}

ACLR with BPTB allograft in patients aged 50 years and older leads to good PROs with significantly increased postoperative activity status at a minimum 2year follow-up

\section{References}

1. Sanders TL, Maradit Kremers H, Bryan AJ, et al. Incidence of anterior cruciate ligament tears and reconstruction: A 21-year population-based study. Am J Sports Med 2016;44:1502-1507.

2. Van de Velde SK, Gill TJ, Li G. Evaluation of kinematics of anterior cruciate ligament-deficient knees with use of advanced imaging techniques, three-dimensional modeling techniques, and robotics. J Bone Joint Surg Am 2009;91:108-114 (suppl 1).

3. Brambilla L, Pulici L, Carimati G, et al. Prevalence of associated lesions in anterior cruciate ligament reconstruction: Correlation with surgical timing and with patient age, sex, and body mass index. Am J Sports Med 2015;43:2966-2973.

4. Stone JA, Perrone GS, Nezwek TA, et al. Delayed ACL reconstruction in patients $>/=40$ years of age is associated with increased risk of medial meniscal injury at 1 year. Am J Sports Med 2019:363546518817749.

5. Andersson D, Samuelsson K, Karlsson J. Treatment of anterior cruciate ligament injuries with special reference to surgical technique and rehabilitation: An assessment of randomized controlled trials. Arthroscopy 2009;25: 653-685.

6. Lewis PB, Parameswaran AD, Rue JP, Bach BR Jr. Systematic review of single-bundle anterior cruciate ligament reconstruction outcomes: A baseline assessment for consideration of double-bundle techniques. Am J Sports Med 2008;36:2028-2036.

7. Samuelsson K, Andersson D, Karlsson J. Treatment of anterior cruciate ligament injuries with special reference to graft type and surgical technique: An assessment of randomized controlled trials. Arthroscopy 2009;25: 1139-1174.

8. Buller LT, Best MJ, Baraga MG, Kaplan LD. Trends in anterior cruciate ligament reconstruction in the United States. Orthop J Sports Med 2015;3:2325967114563664.

9. Arbuthnot JE, Brink RB. The role of anterior cruciate ligament reconstruction in the older patients, 55 years or above. Knee Surg Sports Traumatol Arthrosc 2010;18:73-78.

10. Barber FA, Aziz-Jacobo J, Oro FB. Anterior cruciate ligament reconstruction using patellar tendon allograft: An age-dependent outcome evaluation. Arthroscopy 2010;26: 488-493.

11. Blyth MJ, Gosal HS, Peake WM, Bartlett RJ. Anterior cruciate ligament reconstruction in patients over the age of 50 years: 2- to 8-year follow-up. Knee Surg Sports Traumatol Arthrosc 2003;11:204-211.

12. Osti L, Papalia R, Del Buono A, Leonardi F, Denaro V, Maffulli N. Surgery for ACL deficiency in patients over 50. Knee Surg Sports Traumatol Arthrosc 201 1;19:412-417.

13. Trojani C, Sane JC, Coste JS, Boileau P. Four-strand hamstring tendon autograft for ACL reconstruction in patients aged 50 years or older. Orthop Traumatol Surg Res 2009;95:22-27.

14. Weng CJ, Yeh WL, Hsu KY, et al. Clinical and functional outcomes of anterior cruciate ligament reconstruction with autologous hamstring tendon in patients aged 50 years or older. Arthroscopy 2020;36:558-562.

15. Noyes FR, Butler DL, Grood ES, Zernicke RF, Hefzy MS. Biomechanical analysis of human ligament grafts used in knee-ligament repairs and reconstructions. J Bone Joint Surg Am 1984;66:344-352.

16. Park MJ, Lee MC, Seong SC. A comparative study of the healing of tendon autograft and tendon-bone autograft using patellar tendon in rabbits. Int Orthop 2001;25: 35-39.

17. Muller B, Yabroudi MA, Lynch A, et al. Defining thresholds for the patient acceptable symptom state for the IKDC subjective knee form and KOOS for patients who underwent ACL reconstruction. Am J Sports Med 2016;44: 2820-2826.

18. Briggs KK, Lysholm J, Tegner Y, Rodkey WG, Kocher MS, Steadman JR. The reliability, validity, and responsiveness of the Lysholm score and Tegner activity scale for anterior cruciate ligament injuries of the knee: 25 years later. Am J Sports Med 2009;37:890-897.

19. Corry IS, Webb JM, Clingeleffer AJ, Pinczewski LA. Arthroscopic reconstruction of the anterior cruciate ligament. A comparison of patellar tendon autograft and four-strand hamstring tendon autograft. Am J Sports Med 1999;27:444-454.

20. Bhatia S, Korth K, Van Thiel GS, et al. Effect of reamer design on posteriorization of the tibial tunnel during endoscopic transtibial anterior cruciate ligament reconstruction. Am J Sports Med 2013;41:1282-1289.

21. Rue JP, Ghodadra N, Bach BR Jr. Femoral tunnel placement in single-bundle anterior cruciate ligament reconstruction: A cadaveric study relating transtibial lateralized femoral tunnel position to the anteromedial and posterolateral bundle femoral origins of the anterior cruciate ligament. Am J Sports Med 2008;36: 73-79.

22. Stein DA, Brown H, Bartolozzi AR. Age and ACL reconstruction revisited. Orthopedics 2006;29:533-536.

23. Ventura A, Legnani C, Terzaghi C, Borgo E. Single- and double-bundle anterior cruciate ligament reconstruction in patients aged over 50 years. Arthroscopy 2012;28: 1702-1709.

24. Kuhn MA, Ross G. Allografts in the treatment of anterior cruciate ligament injuries. Sports Med Arthrosc Rev 2007;15: 133-138.

25. Maletis GB, Chen J, Inacio MC, Funahashi TT. Agerelated risk factors for revision anterior cruciate ligament reconstruction: A cohort study of 21,304 patients from the Kaiser Permanente Anterior Cruciate Ligament Registry. Am J Sports Med 2016;44:331-336. 
26. Kaeding CC, Aros B, Pedroza A, et al. Allograft versus autograft anterior cruciate ligament reconstruction: Predictors of failure from a MOON Prospective Longitudinal Cohort. Sports Health 2011;3:73-81.

27. Kaeding CC, Pedroza AD, Reinke EK, Huston LJ, Spindler KP. Risk Factors and predictors of subsequent ACL injury in either knee after ACL reconstruction: Prospective analysis of 2488 primary ACL reconstructions from the MOON cohort. Am J Sports Med 2015;43: 1583-1590.

28. Su CA, Knapik DM, Trivedi NN, Megerian MF, Salata MJ, Voos JE. Femoral interference screw fixation in ACL reconstruction using bone-patellar tendon-bone grafts. JBJS Rev 2020;8:e0066.

29. Maletis GB, Chen J, Inacio MCS, Love RM, Funahashi TT. Increased risk of revision after anterior cruciate ligament reconstruction with soft tissue allografts compared with autografts: Graft processing and time make a difference. Am J Sports Med 2017;45:1837-1844.
30. Dahm DL, Wulf CA, Dajani KA, Dobbs RE, Levy BA, Stuart MA. Reconstruction of the anterior cruciate ligament in patients over 50 years. J Bone Joint Surg $\mathrm{Br}$ 2008;90:1446-1450.

31. Figueroa D, Figueroa F, Calvo R, Vaisman A, Espinoza G, Gili F. Anterior cruciate ligament reconstruction in patients over 50 years of age. Knee 2014;21:1166-1168.

32. Panisset JC, Gonzalez JF, de Lavigne C, et al. ACL reconstruction in over-50 year-olds: Comparative study between prospective series of over-50 year-old and under-40 year-old patients. Orthop Traumatol Surg Res 2019;105:S259-S265.

33. Fayard JM, Wein F, Ollivier M, et al. Factors affecting outcome of ACL reconstruction in over-50-year-olds. Orthop Traumatol Surg Res 2019;105:S247-S251.

34. Costa GG, Grassi A, Perelli S, et al. Age over 50 years is not a contraindication for anterior cruciate ligament reconstruction. Knee Surg Sports Traumatol Arthrosc 2019;27: 3679-3691. 